\title{
Characterization of non-specific lipid transfer protein (nsLtp) gene families in the Brassica napus pangenome reveals abundance variation
}

Yu Liang ${ }^{1 *+}$, Yang Huang ${ }^{2 \dagger}$, Kang Chen ${ }^{3}$, Xiangdong Kong ${ }^{1}$ and Maoteng $\mathrm{Li}^{3^{*}}$

\begin{abstract}
Background: Brassica napus is an important agricultural species, improving stress resistance was one of the main breeding goals at present. Non-specific lipid transfer proteins (nsLTPs) are small, basic proteins which are involved in some biotic or abiotic stress responses. B. napus is susceptible to a variety of fungal diseases, so identify the BnLTPs and their expression in disease responses is very important. The common reference genome of $B$. napus does not contain all B. napus genes because of gene presence/absence variations between individuals. Therefore, it was necessary to search for candidate BnLTP genes in the B. napus pangenome.

Results: In the present study, the BnLTP genes were identified throughout the pangenome, and different BnLTP genes were presented among varieties. Totally, $246 B n L T P$ genes were identified and could be divided into five types $(1,2, C, D$, and $G)$. The classification, phylogenetic reconstruction, chromosome distribution, functional annotation, and gene expression were analyzed. We also identified potential cis-elements that respond to biotic and abiotic stresses in the $2 \mathrm{~kb}$ upstream regions of all BnLTP genes. RNA sequencing analysis showed that the BnLTP genes were involved in the response to Sclerotinia sclerotiorum infection. We identified 32 BnLTPs linked to blackleg resistance quantitative trait locus (QTL).
\end{abstract}

Conclusion: The identification and analysis of LTP genes in the B. napus pangenome could help to elucidate the function of BnLTP family members and provide new information for future molecular breeding in B. napus.

Keywords: nsLTP, Brassica napus, Pangenome, cis-elements, Gene expression, QTL

\section{Background}

Non-specific lipid transfer proteins (nsLTPs) are small, basic proteins that are involved in physiological and biochemical reactions in plant growth, including lipid transport between cell membrane systems, inhibiting

\footnotetext{
*Correspondence: Liangyu@gxnu.edu.cn; limaoteng426@hust.edu.cn ${ }^{\dagger}$ Yu Liang and Yang Huang contributed equally to this work. ${ }^{1}$ Key Laboratory of Ecology of Rare and Endangered Species and Environmental Protection, Guangxi Key Laboratory of Landscape Resources Conservation and Sustainable Utilization in Lijiang River Basin, College of Life Science, Guangxi Normal University, Guilin, China ${ }^{3}$ Department of Biotechnology, College of Life Science and Technology, Huazhong University of Science and Technology, Wuhan, China Full list of author information is available at the end of the article
}

the growth of pathogens and signalling. nsLTPs are lowmolecular-weight cysteine-rich proteins and are widely distributed in the plant kingdom [1]. Their sequences are characterized by an 8-cysteine motif (ECM): C-Xn$\mathrm{C}-\mathrm{Xn}-\mathrm{CC}-\mathrm{Xn}-\mathrm{CXC}-\mathrm{Xn}-\mathrm{C}-\mathrm{Xn}-\mathrm{C}$ [2]. The N-terminal of mature nsLTPs contains a signal peptide typically composed of 21-29 amino acid residues. In addition, some lipid transfer proteins (LTPs) also carried the motifs that add glycosylphosphatidylinositol (GPI)-anchors, which attach proteins to the outside of the plasma membrane [3]. The main feature of the spatial structure of plant nsLTPs is that $\mathrm{H} 1-\mathrm{H} 4$ are connected by four pairs of disulphide bonds that form a stable structure with a hydrophobic hole [4]. The hydrophobic parts of some 
lipids can interact with this hole. To date, many kinds of LTP genes have been isolated in many plants. Boutrot et al. had divided the LTPs into nine types according to sequence similarity [2]. Edstam et al. further classified the plant LTPs into four categories (1, 2, D, G) and several subclasses (C, E, X, etc.) according to the intron position, sequence identity, the distance between cysteine residues in the ECM, and the post-translational modifications [3].

The nsLTP family is ubiquitous and has been studied in many plants, such as in Arabidopsis thaliana, rice, Triticum kiharae and Brassica rapa $[2,5,6]$. nsLTPs are involved in a variety of physiological functions, such as the ability to enhance cell wall extension in tobacco and wheat [7-10]. In addition, Zhao et al. found that overexpression of the OSLTPL $159^{\text {IL } 112}$ could enhance the cold tolerance of rice [11]. Transgenic plants overexpressing the StnsLTP1 gene acquire improved tolerance to multiple abiotic stresses through enhanced activation of antioxidative defence mechanisms [12]. Dhar et al. found an nsLTP gene was down-regulated in response to multiple bacterial pathogens, further research revealed that this $n s L T P$ was required for defence against bacterial and fungal pathogens as well as for normal seedling growth under salinity stress, and it was named as disease-related nonspecific lipid transfer protein 1 (DRN1, 13]. Although its molecular mechanism is not clear, the responses of LTP genes in these studies suggested that nsLTPs are played an important role in plant stress resistance.

Brassica napus is an important oil crop globally. Only a few studies have focused on single genes in nsLTP family, such as BraLTP1, a new nsLTP gene involved in wax production or deposition, it also has direct or indirect effects on cell division and flower development [13]. Another study found that a new nsLTP gene BraLTP2 might be involved in trichome development and the accumulation of secondary metabolites [14]. However, there has been no genome-wide analysis of nsLTP family in B. napus. After the reference genome of rapeseed cultivar 'Darmorbzh' was published in 2014, a higher-quality genome of the cultivar 'ZS11' was released in 2017 [15, 16]. However, owing to the existence of structural variations, especially the presence/absence variations (PAV), the genome of a single cultivar cannot completely cover the entire gene catalogue. Pangenomes have been constructed for many species to obtain more genetic data resources, such as soybean, maize, rice, tomato [17-21]. In 2019, Song et al. constructed a high-quality pangenome of $B$. napus by using eight $B$. napus varieties [22, 23]. In the present study, the nsLTP gene identification, characterization, and expression analysis were conducted by whole-pangenome, and a total of 246 nsLTP family genes were identified, and which could be classified into five types: $1,2, \mathrm{C}$, $\mathrm{D}, \mathrm{G}$. We classified the nsLTPs belonging to the reference genome and non-reference regions, and present a more comprehensive understanding of nsLTP distribution in different varieties. The phylogeny, $\mathrm{Ka} / \mathrm{Ks}$, GO enrichment and expression of the nsLTP family were also analyzed. These results lay a foundation for further studies on the function of the LTP family in B. napus.

\section{Method}

Identification of nsLTP genes in the $B$. napus pangenome

The pangenome sequence was downloaded from the B. napus pangenome information resource (http://cbi. hzau.edu.cn/bnapus/) [22]. The coding sequence (CDS) and protein sequences of the pangenome were obtained by gffread v0.12.7 with the pangenome sequences and annotations [24]. NsLTP sequences of $A$. thaliana were downloaded from TAIR (http://www.arabidopsis.org). The homologous nsLTPs genes between $A$. thaliana and $B$. napus were identified by comparing the CDS sequences to the $A$. thaliana nsLTPs sequences with blastx v 2.2.26 using a threshold of 1E-5. The Tryp alpha_amyl.hmm and LTP_2.hmm files in pfam [25] were downloaded, and hmmscan v3.3.1 was used to identify candidate LTP family genes with a threshold of $1 \mathrm{E}-5$. After finding the candidate nsLTPs in two ways, the protein sequences were submitted to the conserved domain database (CDD) [26] and InterProScan (https:// www.ebi.ac.uk/interpro/search/sequence/) to check for the presence of LTP domains.

An in-house perl script was then used to check for the presence of the ECM structure in the candidate LTP, and sequences with no ECM structure were removed. Then, we used SignalP v4.1 to predict the location of the signal peptides [27]. Sequences without signal peptides were also removed.

\section{NsLTP sequence analysis}

Two prediction tools, PredGPI (http://gpcr2.bioco mp.unibo.it/gpipe/pred.htm) and GPI-SOM (http:// gpi.unibe.ch/), were used to determine the presence of C-terminal GPI-anchor sites. Sequences with GPIanchor sites were classified as category G, and the others were classified according to Edstam et al. [3]. $A$. thaliana nsLTP sequences were downloaded from TAIR, and B. napus LTP sequences were used to construct a phylogenetic tree. The multiple sequence alignment tool MEGA7 was used to generate a phylogenetic tree by the neighbour-joining method. MEME v2.0.0 [28] was used to analyse the conserved motifs in the $B$. napus nsLTP genes, with the highest number of motifs set to 10 . Gene structures were visualized using GSDS $2.0[29]$. 


\section{Gene duplication analysis, synonymous} and non-synonymous substitution rates estimation The duplication of genes can be divided into two types: tandem duplication and segmental duplication. The criterion for tandem duplication of LTP gene is that the physical distance of two genes on the chromosome should fall within $50 \mathrm{~kb}$ [30].

Segmental duplication refers to the case where the two genes are physically far away on the chromosome, but are still homologous. The criteria are as follows: (1) the region of overlap between homologous genes accounts for more than $80 \%$ of the total length of longer genes and (2) the sequence similarity is more than $80 \%$. Synonymous and non-synonymous substitution rates of duplicated genes were calculated by TBtools v1.068 [31].

\section{Cis-element analysis of the promoter regions of the nsLTP genes}

In this study, in-house perl script was used to extract the upstream $2 \mathrm{~kb}$ sequence of BnLTP genes. The binding site prediction tool (http://plantregmap.gao-lab.org/ binding_site_prediction.php in plantregmap) was used to predict TF binding sites in the $2 \mathrm{~kb}$ upstream sequence of BnLTPs.

\section{Gene ontology (GO) annotation and enrichment analysis}

All BnLTP sequences were compared with sequences in the uniprot database using blastp with a threshold of 1E-5. Using the Retrieve/ID mapping tool (https://www. uniprot.org/uploadlists/), uniprot IDs were converted to GO IDs to perform GO annotation for BnLTPs. AgriGO (http://bioinfo.cau.edu.cn/agriGO/analysis.php) was used for GO enrichment analysis. All genes of the Brassica napus pan-genome were used as the background gene set.

\section{RNA-seq data analysis}

The RNA-seq data were downloaded from NCBI with the SRA accession number SRP053361(https://www.ncbi. nlm.nih.gov/sra/?term=SRP053361, 33]. Fastq-dump of SRA Toolkit v2.10.0 (http://www.ncbi.nlm.nih.gov/
Traces/sra/sra.cgi?view=toolkit_doc $\& \mathrm{f}=$ fastq-dump) was used to convert the downloaded SRA file to FASTQ Format file. Fastp v0.20.1 was used to remove adapters and low-quality sequences in the original data.

The RNA-seq clean data of each sample were aligned to the Brassica napus pan-genome using HiSAT2 v2.1.0. The FPKM (Fragments Per Kilobase of transcript per Million mapped reads) values of genes in all samples were calculated as the expression level. The DESeq2 v1.32.0 [32] was used for differential expression analysis, and unigenes with $\mid \log 2$ ratio $\mid \geq 1$ and $\mathrm{q}<0.05$ as a cut-off for significant differential expression were selected. The transformed $\log 2(\mathrm{FPKM}+1)$ values were used to generate the heatmap using $R$ (version 4.0.3) with the ComplexHeatmap package (version2.6.2, https://bioconduct or.org/packages/release/bioc/html/ComplexHeatmap. html).

\section{Linking known QTL and BnLTP genes}

Gene and QTL region overlap was determined using bedtools v2.16.2 intersect [33]. Known blackleg resistancelinked QTL were collected from the literature [34-38]. Waterfall plots were drawn using Variant Effect Predictor v99 [39], GenVisR v1.11.3 [40], vcftools v0.1.16 [41].

\section{Results}

Pangenome-wide identification and distribution of nsLTPs in B. napus

Song et al. sequenced and assembled high-quality genomes of eight varieties: ZS11, Gangan (GG), Zheyou7 (ZY), Shengli (SL), Tapidor (TA), Quinta (QU), Westar (WT), and No2127 (NO) [22], combined with the genome of 'Darmor-bzh' [15], and the pangenome of $B$. napus was constructed. Totally, 246 BnLTP sequences with an ECM domain were obtained, including $115 \mathrm{G}$ typeGPI-anchored nsLTPs (detected by GPI-SOM and PredGPI) and 1, 2, C and D types of 29, 50, 4 and 48 without a GPI-anchored nsLTPs (the type-specific cysteine spacing patterns suggested by Edstam et al.) (Table 1).

In the pangenome-wide search for nsLTP genes, $B n L T P$ genes were identified on all chromosomes in the

Table 1 Characteristics of different types of B. napus non-specific lipid transfer proteins (nsLTPs)

\begin{tabular}{|c|c|c|c|c|c|c|c|c|c|}
\hline \multirow{2}{*}{$\begin{array}{l}\text { Type } \\
1\end{array}$} & \multirow{2}{*}{$\begin{array}{l}\begin{array}{l}\text { Number of } \\
\text { members }\end{array} \\
29\end{array}$} & \multirow{2}{*}{$\begin{array}{l}\text { GPI-anchor } \\
\text { NO }\end{array}$} & \multicolumn{7}{|c|}{ Cysteine Spacing Pattern } \\
\hline & & & C & X9 & $\mathrm{C} 13,14$ & $\mathrm{CC}$ & $X 19,20$ & CXC & $\times 19,21-24$ \\
\hline 2 & 50 & NO & C & X7 & $\mathrm{C} 13,14$ & $\mathrm{CC}$ & $x_{8}$ & $C X C$ & $X 10,19,21,23,25$ \\
\hline C & 4 & NO & C & X9 & $\mathrm{C} 16$ & $\mathrm{CC}$ & X9 & $C X C$ & $\mathrm{X} 12$ \\
\hline D & 48 & NO & C & $x 9,10,13$ & $\mathrm{C} 12,14-19$ & $\mathrm{CC}$ & $x 9,12,13$ & $C X C$ & $X 19,22,24,26$ \\
\hline G & 114 & YES & C & $x 6,7,9,10,11,13$ & $\mathrm{C} 11,13-18$ & $\mathrm{CC}$ & $x 8,9,12-14,19$ & $C X C$ & $X 19,22-26,19$ \\
\hline
\end{tabular}


reference genome of ZS11 (Table S1), in which, 197 were located in the reference genome of ZS11 and 49 were located in non-reference regions. It was revealed that the BnLTPs were unevenly distributed on different chromosomes, $\mathrm{C} 02$ and $\mathrm{C} 03$ were with 18 BnLTPs, and only two LTPs were located on A04. According to PAV data, the LTP family genes identified in non-reference genomes were distributed across 13 chromosomes and A09 was with the most numbers (Fig. 1). The non-reference regions contained 1 (1), 2 (7), D (13) and G (23) types of BnLTPs with the number of 1, 7, 13 and 23, respectively. According to the PAV information, the BnLTPs were not evenly distributed in all varieties (Table 2), and the variety GG had the most of 13 BnLTP genes, otherwise, only 1 was observed in QU, it indicated that BnLTP genes may be lost in QU and duplicated in GG during domestication. The above results also suggested that when analysing the nsLTP gene family should not only focus on the reference genome.

\section{Phylogenetic analysis and BnLTP sequence features of the B. napus}

The neighbour-joining method was used to construct a phylogenetic tree based on the nsLTP sequences of $B$.

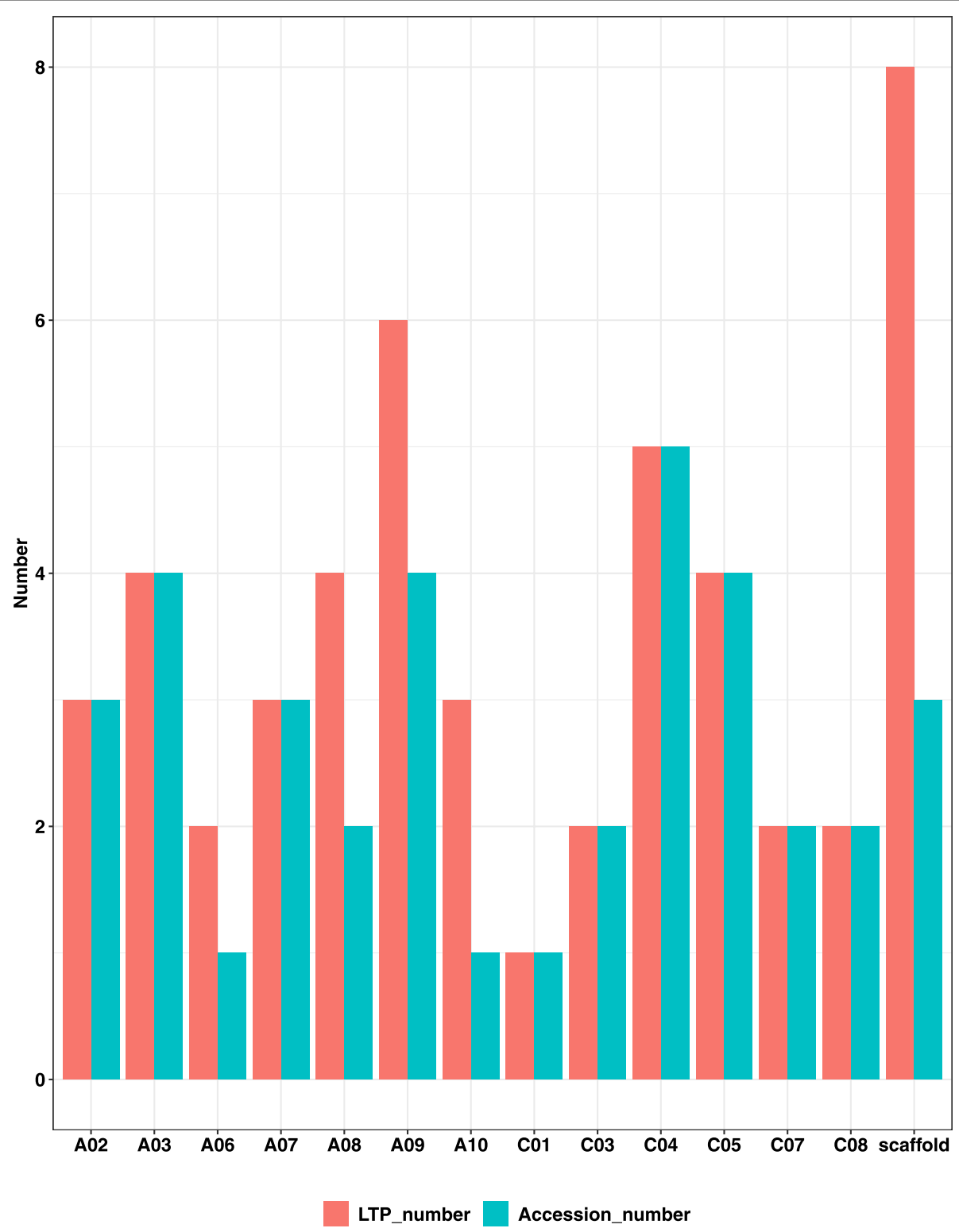

Fig. 1 Distribution of BnLTPs in presence/absence variation regions of the chromosomes in B. napus 
Table 2 Count of BnLTP genes per variety and per BnLTP type

\begin{tabular}{llllllll}
\hline Type & D & GG & NO & QU & SL & TA & WE \\
\hline 1 & 0 & 1 & 1 & 0 & 0 & 1 & 1 \\
2 & 2 & 2 & 2 & 0 & 1 & 0 & 0 \\
C & 0 & 0 & 0 & 0 & 0 & 0 & 0 \\
D & 1 & 4 & 2 & 1 & 2 & 1 & 0 \\
G & 1 & 6 & 4 & & & 1 & 2 \\
\hline
\end{tabular}

napus and $A$. thaliana. The branching of the tree is consistent in general with the classification into the main structural types and indicates that $n s L T P$ genes in each clade were originated from a common ancestor (Fig. 2), further analysis revealed that the types1, D, and G have two, five and eight sub-branches, respectively. According to Fig. 2, the typeD and G BnLTPs are closely related, since many sub-branches of typeD and $\mathrm{G}$ are very close in the phylogenetic tree.

ECM sequence logos were constructed for each BnLTP type to explore which amino acid residues were conserved between adjacent cysteine residues. Figure 3 shows that type1, 2 , and $C$ are more conservative than that of typeD. The CXC structure can affect cysteine pairing and influence protein folding. Among all the BnLTP sequences, there were 12 different amino acid residues in the CXC structure: A, E, F, G, I, K, L, M, Q, R, V, and Y. Hydrophobic residue $L$ was the most common residue in type2, D, and G. In type1, the proportions of R, K, Q, and $\mathrm{E}$ residues were similar.

\section{Duplication patterns and synteny analysis of the BnLTP genes}

Gene duplication is considered to be a driving force in evolution and an important factor to increase genome complexity. B. napus is an allotetraploid formed by natural interspecific hybridization and chromosome doubling between B. oleracea (CoCo, $2 n=18$ ) and B. rapa (ArAr, $2 n=20,16]$. Therefore, there are usually two or more duplicated gene sequences in the genome of B. napus. Segmental and tandem duplications are considered to be the main reason why the gene family is expanded. In the present study, 22 pairs of tandem duplications were identified (Table S2), including two pairs in GG, three in $\mathrm{SL}$, and 17 in ZS11. Among them, the A10 chromosome of Shengli had the highest number of tandem duplication events. It was revealed that three pairs of tandemly duplicated BnLTP genes (BnLTPD.43, BnLTPD.44, $B n L T P D .45)$ were all in the same duplicated gene blocks. Segmental duplications were identified by blastp with the requirement that there was $>80 \%$ overlap with the longer sequence, $>80 \%$ sequence similarity, and the repeated pairs are counted only once. A total of 339 segmental duplications BnLTP pairs (Table S3) were identified, including of 4 genes in D, 2 in TA, 1 in QU, 9 in NO, 3 in WE, 13 in GG, 7 in ZY and 7 in SL. Among them, the C03 chromosome of ZS11 had the highest number of segmental duplication events. We also analyzed the synteny of the BnLTP genes between the reference genome and non-reference regions (Fig. 4). Many variety-specific $B n L T P$ genes were with a syntenic relationship with the BnLTP genes on the reference genome, such as 13 $B n L T P$ in Gangan have syntenic relationship with the BnLTP genes on chromosomes A06, A07, A08 and A09 of the reference genome, and 11 Gangan-specific BnLTPs have a syntenic relationship with the $B n L T P$ on the reference chromosomes of C04, C05, C07, C08 and C09 of B. napus $\mathrm{C}$ genome. In the varieties Quinta and Tapidor, only one specific gene maintained a syntenic relationship with $B n L T P$ on the reference genome. This indicated that $B n L T P$ s have different duplication patterns in different varieties.

\section{Cis-regulatory factors and GO analysis of the LTP gene}

Analysis of the $2 \mathrm{~kb}$ upstream regions (from the translation start site) of BnLTP genes could reveal the presence of various regulatory elements that are associated with the development and abiotic/biotic stress signalling. It was revealed that the ethylene-responsive factor (ERF) binding sites account for the largest proportion of sites in the upstream regions of the genes in the reference genome and non-reference region (Fig. 5), these sites are involved in hormonal signal transduction, response to biotic and abiotic stresses, regulation of metabolism, and developmental processes in various plant species. Weidong Wang et al. found that the upstream of LEA gene family in tea species is regulated by ERF, MYB and bZIP and bHLH transcription factor [42], which shows that the genes regulated by AP2/ERFs is a common phenomenon. Some other regulatory elements involved in the regulation of growth and development processes, such as the Dof (DNA binding with one finger) binding site, were also enriched in BnLTPs. The pathogen- and viral infection-responsive cis-regulatory elements included of the TF binding sites NAM, ATAF, and CUC (NAC) were also identified. Other regulatory elements, such as binding 


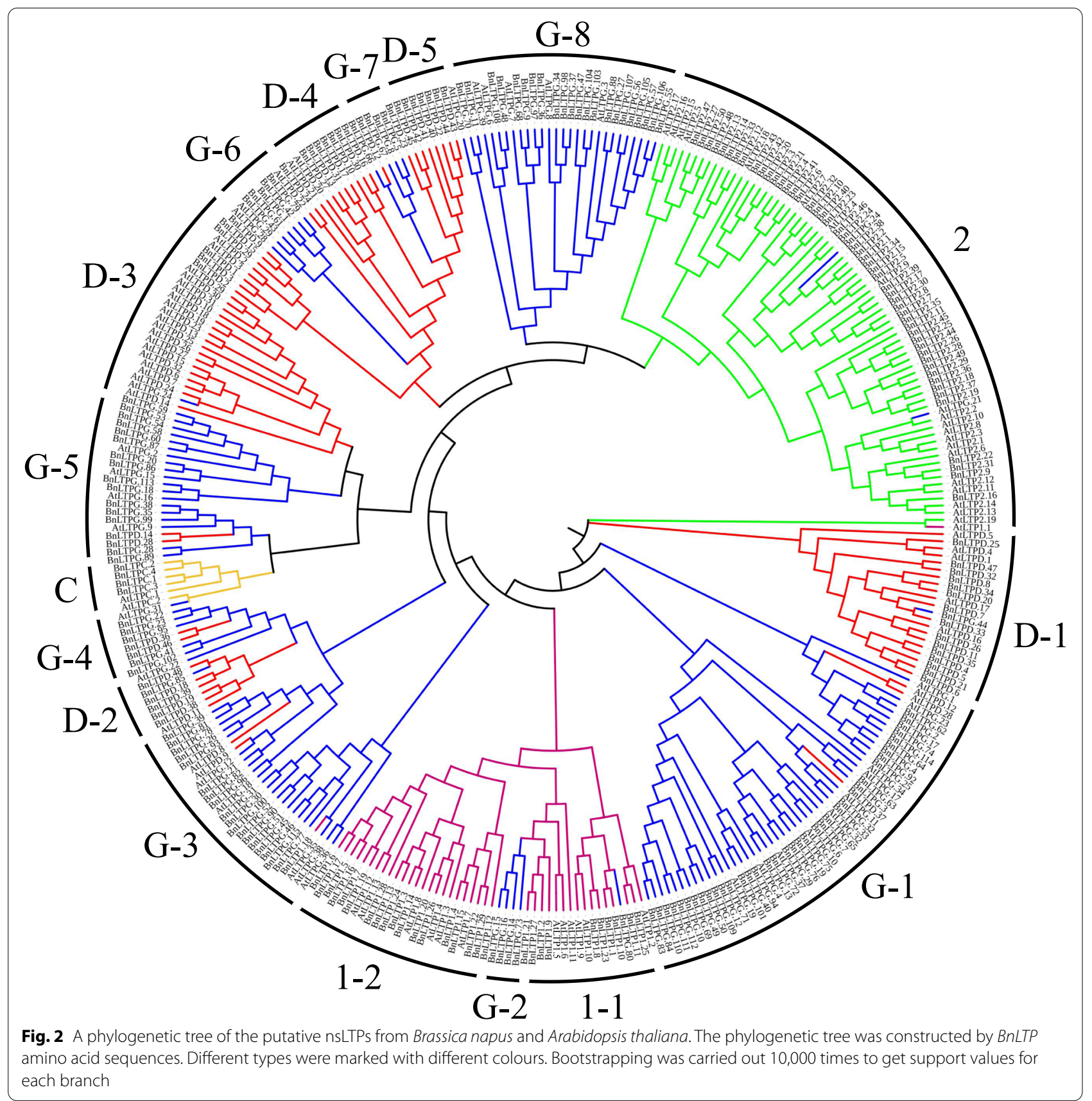

sites for $\mathrm{C} 2 \mathrm{H} 2$ zinc finger transcription factor (involved in the occurrence of plant leaves and the regulation of floral organs) and lateral organ boundaries domain (LBD, involved in the development and formation of plant lateral organs), also existed.

To further understand the function of LTP in organisms, GO annotation and enrichment analysis of LTP genes were performed. Figure $6 \mathrm{~A}$ shows that binding (GO:0005488) was the most abundant GO term that LTPs were identified as being involved in, which is consistent with the function of BnLTP. In the GO function enrichment analysis, significant enrichment was observed for a large number of GO terms related to binding and transport, such as lipid binding, fatty acid binding, monocarboxylic acid binding, lipid transport, and organic substance transport. Meanwhile, systemic acquired resistance and salicylic acid-mediated signalling pathways were also significantly enriched, which reveals that the nsLTP gene family was involved in biotic and abiotic stress responses. (Fig. 6B, C, D). 
Liang et al. BMC Plant Biology

(2022) 22:21

Page 7 of 14

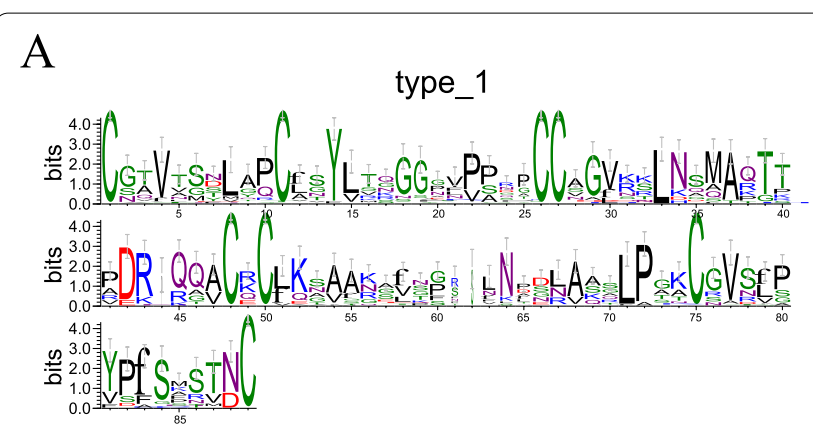

B

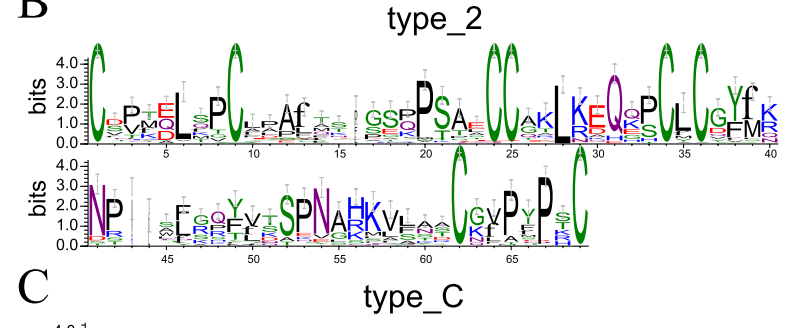

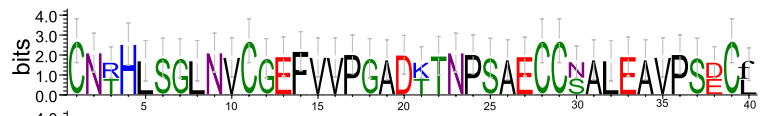

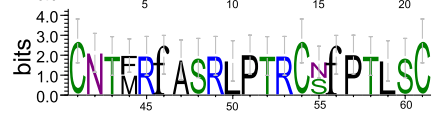

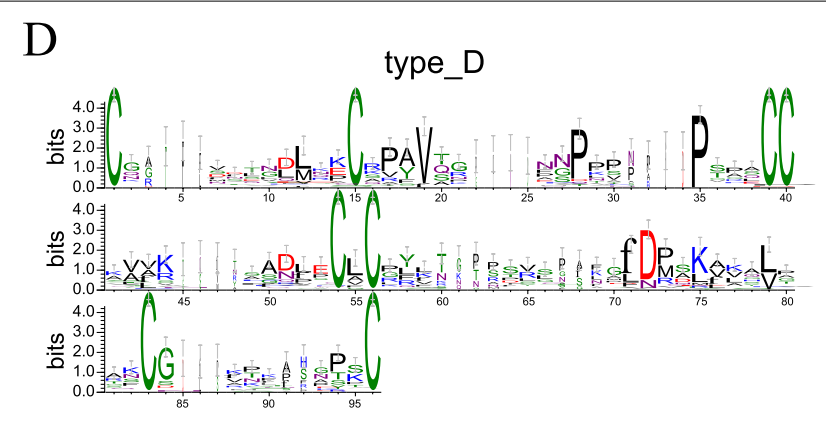

E

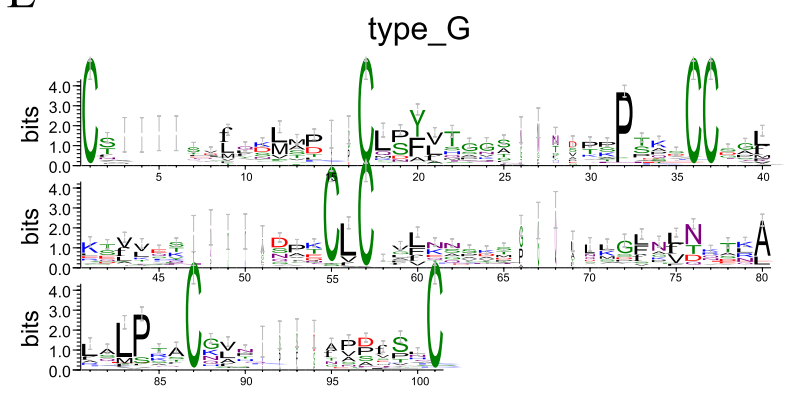

Fig. 3 Sequence logo plots of an 8-cysteine motif (ECM) region sequences for each Brassica apus lipid transfer proteins type. Larger amino acid residues in the picture indicate higher sequence conservation level
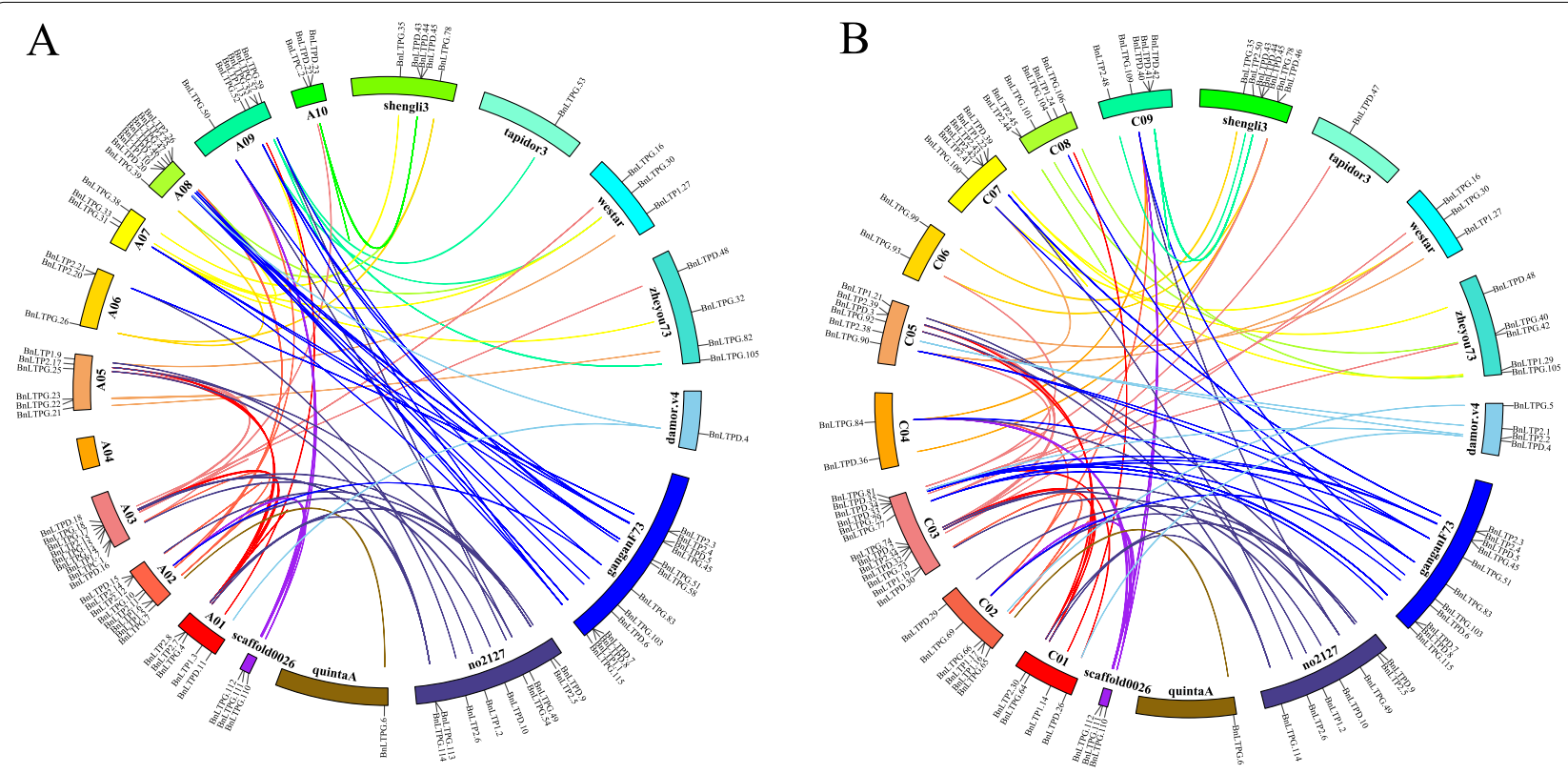

Fig. 4 A, Distribution and syntenic relationship of the BnLTP genes in the B. napus A genome and pan-genome additional contigs. B, Distribution and syntenic relationship of the BnLTP genes in the B. apus $C$ genome and pan-genome additional contigs 

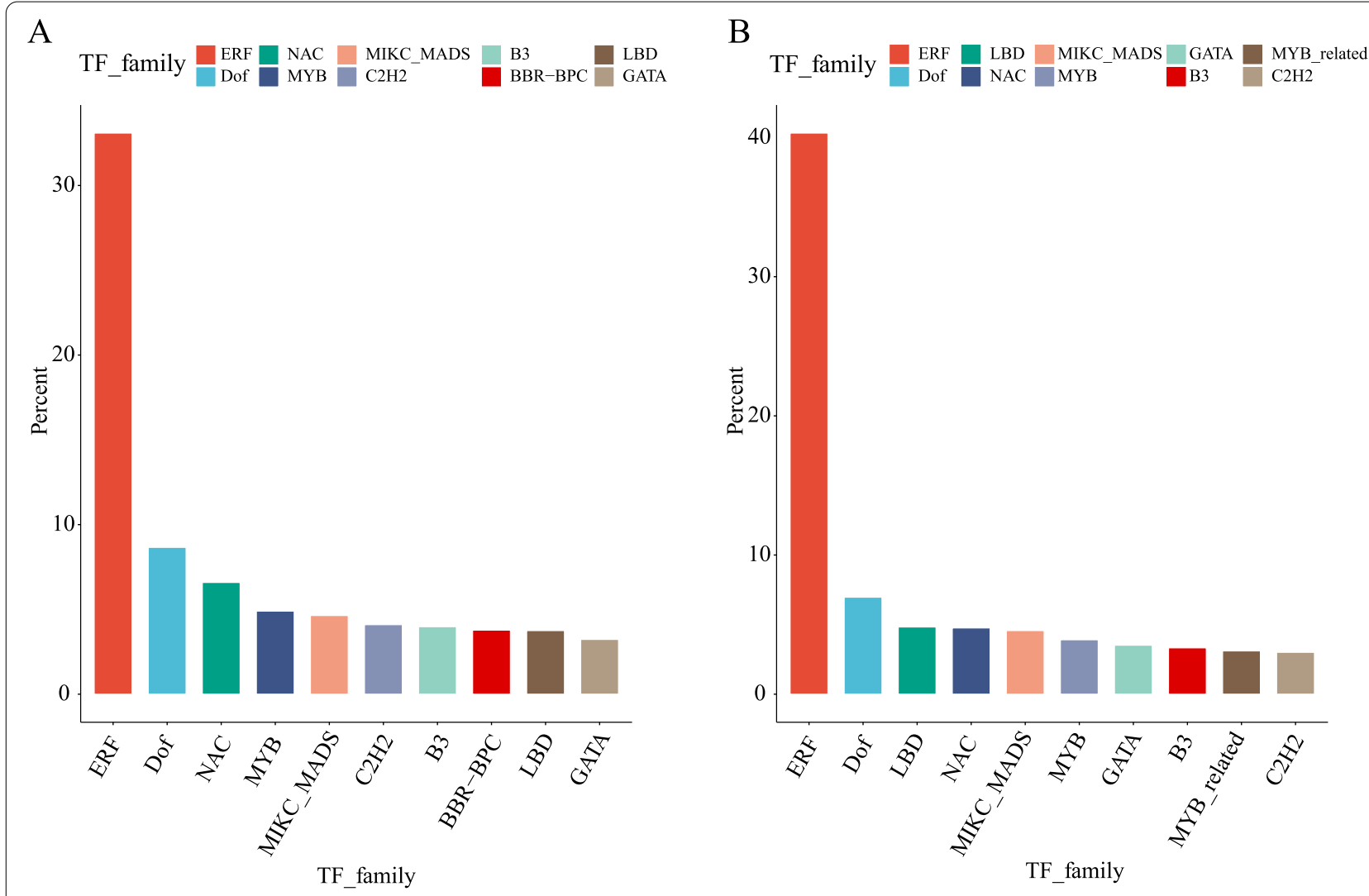

Fig. 5 The top 10 cis-elements identified in $2 \mathrm{~kb}$ upstream regions of BnLTP genes. A Upstream regions of the genes in the reference genome. $\mathbf{B}$ Upstream regions of the genes in the pan-genome additional contigs.

\section{The expression of BnLTP genes that response to Sclerotinia sclerotiorum}

Sclerotinia stem rot, caused by S. sclerotiorum, is one of the most destructive diseases in rapeseed. Wu et al. studied the transcriptional response of rapeseed under the resistant line (R-line) and a susceptible line (S-line) [43]. Compared to the mock-inoculated samples, only two BnLTPs were differentially expressed in R-line in $24 \mathrm{~h}$ after infection, while in S-line, $16 \mathrm{BnLTP}$ genes were differentially expressed (Fig. 7A) in $24 \mathrm{~h}$ after infection. After 48 and $96 \mathrm{~h}$ of infection, the $\mathrm{R}$ and $\mathrm{S}$ lines showed similar numbers of differential expression genes. However, according to Fig. 7A and B, the response of BnLTPS to inoculated S. sclerotiorum was different at different periods in the two lines. In the R-line, there were no common differentially expressed $B n L T P$ genes between the three periods, and $20 B n L T P$ genes were only found in $96 \mathrm{~h}$ after infection. In the $\mathrm{S}$-line, only eight $B n L T P$ genes were found in all three periods, and 16 BnLTP genes were only in $96 \mathrm{~h}$ after infection. The two lines were compared at different infection stages, and there were 44, 17, and 18 differentially expressed BnLTP genes 24, 48, and $96 \mathrm{~h}$ after infection (Fig. 7C), respectively, indicating that the response of $B n L T P$ genes in the two varieties was the most different in the early stage of infection.

$\log ^{2}($ FPKM+1) values of the genes were used to plot the heatmap (Fig. 7D), it shows that some typeG BnLTP genes exhibited low expressions, but exhibited most differential expression. There were fewer type1 BnLTP genes than type2 BnLTP genes in the pangenome, but more differentially expressed type1 BnLTP genes than type2 $B n L T P$ genes. Figure 7D shows the expression level of each differentially expressed BnLTP gene in the different samples. The expression of BnLTPG.7 and BnLTPG.72 genes was lower in the R-line than in the $\mathrm{S}$-line.

\section{Linking BnLTP genes to known QTL for blackleg resistance} To further assess possible biological functions of BnLTPs, we compared the position of BnLTPs with known QTL for blackleg resistance [44]. Positions were predicted from known genomic regions of seven loci in the ZS11 assembly. The QTL name and references are shown in Table 3. These QTLs covered between $5.3 \mathrm{Mb}$ and $30.5 \mathrm{Mb}$ on three pseudomolecules (Rlm1, Rlm3, Rlm4, Rlm7 and Rlm9 were located on A07, as well as LepR1 


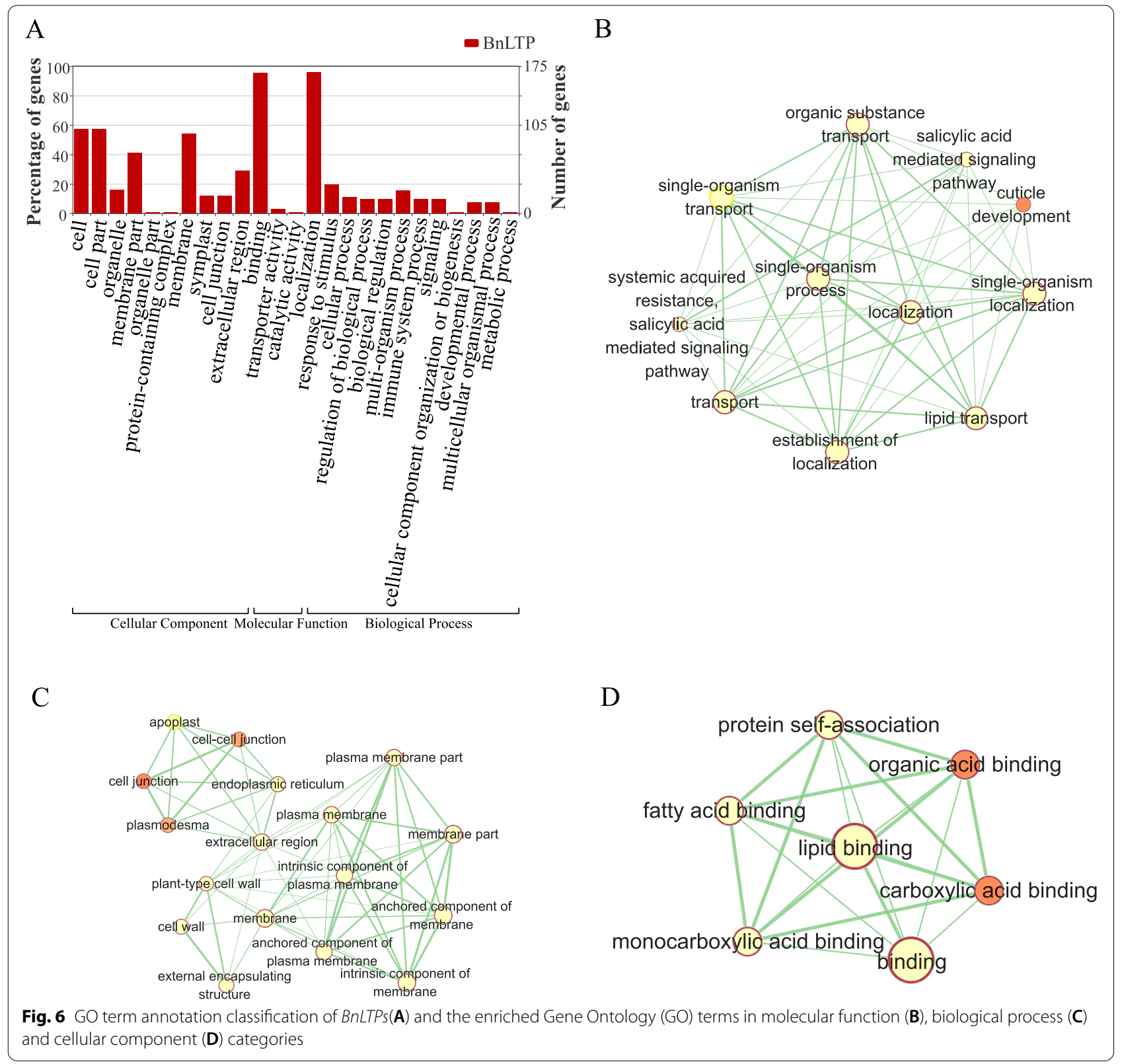

and LepR2 was located on A02 and A10 respectively). Furthermore, the mapping of R $\operatorname{lm} 4$ covered the greatest number of BnLTPs which 1689 consist of 4 core and 4 variable genes. Rlm7 and Rlm9 loci contained only one variable $B n L T P$. The $R \operatorname{lm} 1$ was the narrowest QTL but contained 5 BnLTPS which number was in the medium of all loci.

Song et al. combined rape resequencing data produced by several studies, then a large number of variations were identified [23]. Based on the mutation information, two waterfall plots of the blackleg-linked QTL (LepR1 locus, Rlm3 locus and Rlm4 locus) were produced to show the mutational load of BnLTPs located within the QTL candidate regions. We filtered $B n L T P s$ without SNP and individuals without mutation in BnLTPs and finally showed the mutation load of 773 individuals in Rlm 3 and Rlm4 loci as well as 1283 individuals in LepR1. Moreover, the loci of Rlm3 and Rlm4 covered the same BnLTPs thus their waterfall plots are identical (Fig. 8). As shown in the waterfall plot of Rlm3 and Rlm4 loci, the maximum and minimum mutation percentages were related to BnLTPG3.1 and BnLTP2.22, respectively (Fig. 8A). BnLTP2.11 exhibited the highest mutation in all BnLTPs located on LepR1 locus, and 

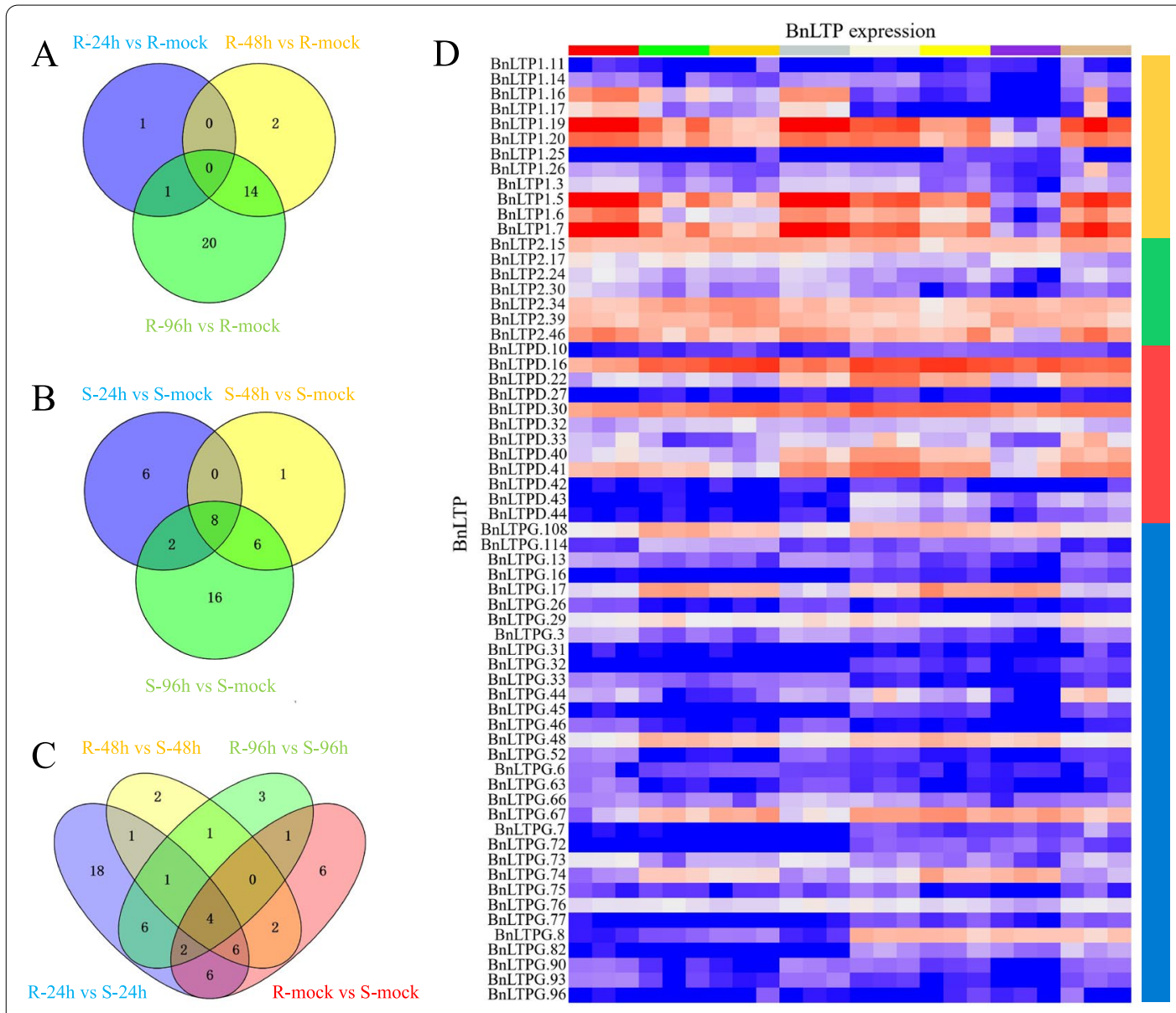

Type 1

Type 2

Fig. 7 Transcriptome analysis of BnLTP responses to Sclerotinia sclerotiorum. A, B, C Venn Diagrams of overlapping and specific differentially expressed BnLTP genes for different comparisons. D Heatmap of the expression of the differentially expressed BnLTP genes

Table 3 BnLTPs underlying reported QTL for blackleg in the ZS11 assembly

\begin{tabular}{|c|c|c|c|c|c|c|c|}
\hline Locus & Resistance & Publication & Pseudomolecule & $\begin{array}{l}\text { Start } \\
\text { (bp) }\end{array}$ & $\begin{array}{l}\text { End } \\
\text { (bp) }\end{array}$ & $\begin{array}{l}\text { Length } \\
\text { (bp) }\end{array}$ & Core genes percentage \\
\hline RIm1 & blackleg & $\begin{array}{l}\text { Delourme et al., } 2004 \\
\text { Raman et al., 2012b }\end{array}$ & $\mathrm{A} 07$ & $25,515,316$ & $30,891,516$ & $5,376,200$ & $\begin{array}{l}3 \text { core, } 2 \text { variable } \\
\text { (60\% core) }\end{array}$ \\
\hline RIm3 & blackleg & $\begin{array}{l}\text { Delourme et al., } 2004 \\
\text { Leflon et al., } 2007\end{array}$ & A07 & $11,368,284$ & $29,211,062$ & $17,842,778$ & $\begin{array}{l}1 \text { core, } 3 \text { variable } \\
\text { (25\% core) }\end{array}$ \\
\hline RIm4 & blackleg & $\begin{array}{l}\text { Raman et al., 2012a } \\
\text { Tang and Zhao, } 2015\end{array}$ & A07 & $1,512,732$ & $32,016,319$ & $30,503,587$ & $\begin{array}{l}4 \text { core, } 4 \text { variable } \\
\text { (50\% core) }\end{array}$ \\
\hline$R / m 7$ & blackleg & Larkan et al., 2016 & $\mathrm{~A} 07$ & $15,452,927$ & $23,156,986$ & $7,704,059$ & $\begin{array}{l}0 \text { core, } 1 \text { variable } \\
\text { (0\% core) }\end{array}$ \\
\hline R/m9 & blackleg & Delourme et al., 2004 & A07 & $17,146,407$ & $22,717,328$ & $5,570,921$ & 0 core, 1 variable (0\% core) \\
\hline LepR1 & blackleg & Larkan et al., 2016 & A02 & $10,497,184$ & $23,959,094$ & $13,461,910$ & 1 core, 6 variable ( $14.29 \%$ core) \\
\hline LepR2 & blackleg & Larkan et al., 2016 & $\mathrm{~A} 10$ & 253,009 & $20,875,594$ & $20,622,585$ & $\begin{array}{l}3 \text { core, } 3 \text { variable } \\
\text { (50\% core) }\end{array}$ \\
\hline
\end{tabular}




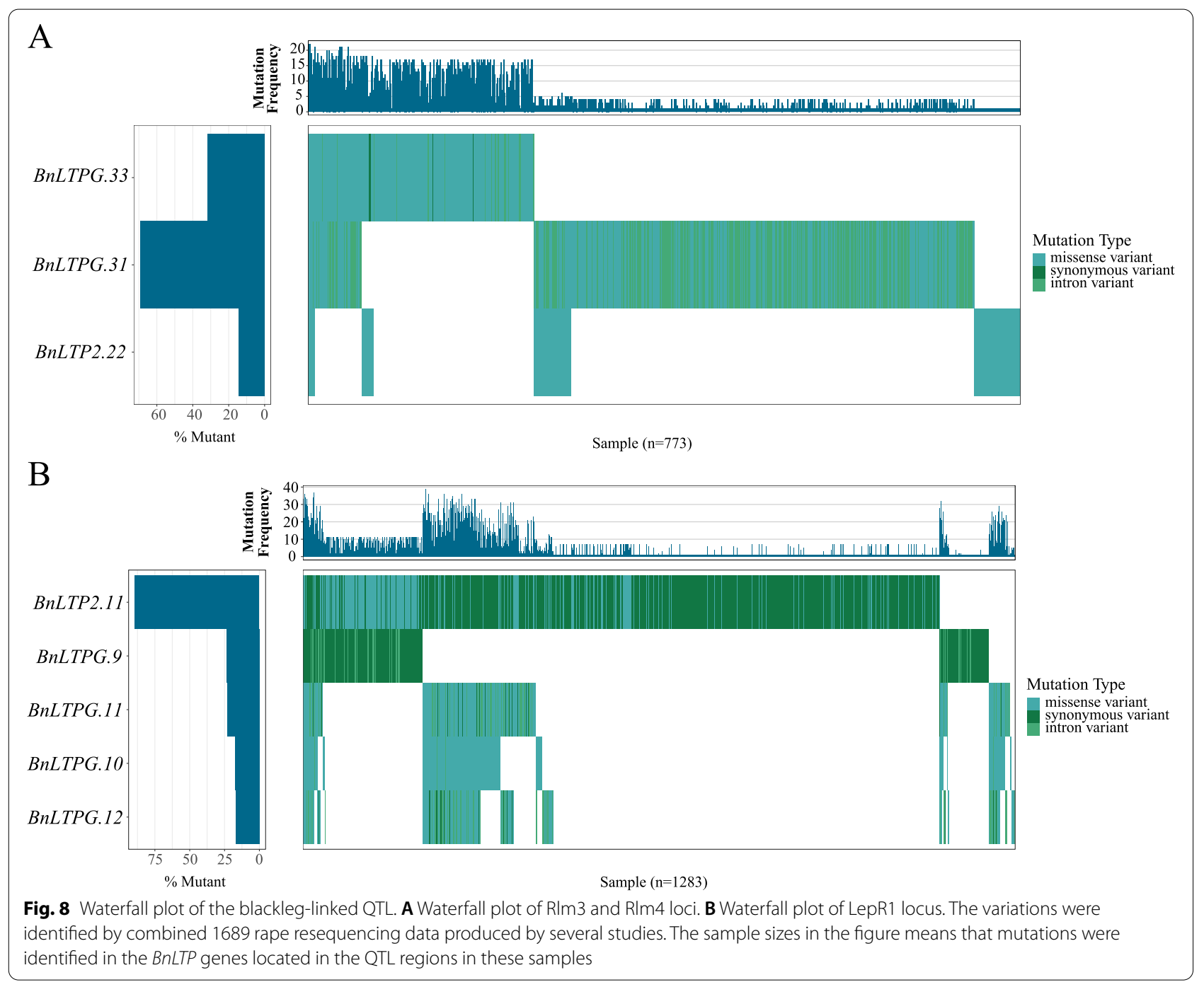

mainly observed synonymous variant which has very few low impacts.

\section{Discussion}

In this study, 246 BnLTP genes were identified, including of 29 type1, 50 type2, four typeC, 48 typeD, and 114 type $G$ in the entire pangenome of rapeseed. In addition to the 197 BnLTPs found in the reference genome, another 49 BnLTPs in the non-reference region of the non-reference sequences also existed. In the study of Hurgobin et al. [45], 53 synthetic and nonsynthetic accessions were used to generate the first $B$. napus pangenome. 13,631 new genes were predicted in the pangenome. We compared the 13,631 genes with the pan-genome published by Song et al. [22] using the software BLASTN with the parameters'- e 1emur10' and'- F F 'and a sequence identity of $>98 \%$. Finally a new nSLTP gene was identified uniquely in the 13,631 genes. It indicates that more members of gene family may be found in the genomic data resources of more varieties, and more nsLTP genes can be discovered on the basis of this study in the future.

In different cultivated species, the distribution of $B n L T P$ s in the non-reference region was not balanced, which indicated that the different varieties underwent different forms and levels of selection during domestication. In the reference genome, the identified BnLTPs were unevenly distributed across the genome. The uneven distribution of BnLTPs might be due to recent BnLTP gene tandem duplications, segmental duplications and dosage compensation, which is critical for development and phenotypic characteristics [44]. The results show that the order and proximity of genes of the gene family are important to the functional nature of these genes [44]. 
In $B$. oleracea, Bayer et al. revealed that the single nucleotide polymorphisms (SNPs) and presence-absence variation (PAV) of $B$. oleracea have experienced different selection pressures for different resistance gene analog (RGA) classes [46]. Dolatabadian et al. identified 1749 RGAs in the B. napus pangenome, 996 of which are core, 753 are variable, and 368 are not present in the reference genome [45]. The present study and the above mentioned two pangenome studies showed that it is necessary to identify candidate genes in the pangenome for breeding work, as it can avoid the disadvantage of having incomplete information on a single reference genome. Among the identified BnLTPs, 22 and 339 pairs were tandem duplicates and segmental duplicates, respectively, it indicated that the gene duplications played an important role in the expansion and evolution of $n S L T P$ residence in rapeseed. This phenomenon is not only found in rape, but also in other species, e.g., A. thaliana, turnip, rice, and maize $[2,6,47]$. In this study, the phylogenetic relationships of nsLTPs between B. napus and the A. thaliana showed a high degree of consistency in the same type between the two species.

Previous studies have shown that the nsLTPs are involved in a variety of biotic and abiotic stress responses $[5,48]$.Odintsova et al. speculated that nsLTPs are involved in the activation of pathogen defences [5]. Their mechanisms of action might be due either directly to the antimicrobial or lipid-transferring activity or indirectly as signalling molecules [5]. Another study found that overexpression of AtLTP4.4 could protect against the oxidative stress that induced by trichothecene by increasing the glutathione-based antioxidant defence effect [49]. These results suggested that the $n s L T P$ family might play an important biological role in plant responses to pathogen infection. In order to study the role of BnLTP in the response to $S$. sclerotiorum, we analyzed the sequencing data (accession no. SRP053361) of two rape varieties (a resistant line and a susceptible line) under S. sclerotiorum infection for 24, 48, and $96 \mathrm{~h}$ [43], it was revealed that the sixty-one BnLTPs were identified to be differentially expressed in different samples, which also indicated that different BnLTP types have different expression patterns (Fig. 7D).

The experiment in vitro showed that nsLTPs are involved in many plant disease resistance. In some in vitro studies, heterologous expression and purification of nsLTPs showed resistance to a variety of plant pathogens [50, 51]. nsLTP is a plant disease-related protein (PRs) and the expression level increased with the inducing of pathogenic bacteria as well as inhibit the reproduction of pathogenic bacteria and induce the resistance-related response in plants [51]. By some biochemical methods and overexpression or knockout experiments, it was demonstrated that some nsLTPs are playing important role in resisting the invasion of pathogens [52-54]. For example, Patar et al. overexpressed the onion (Allium cepa) Ace-AMP1 in rice, which improved the resistance of rice blast, sheath blight and parabola without changing other agronomic traits [50]. Overexpressed wheat Ltp 3F1 in tobacco improved plant resistance to fungal diseases [51]. Inhibiting the expression of CALTPI and CALTPII in pepper, transgenic plants showed susceptibility to bacterial diseases. After overexpressing CALTPI and CALTPII in tobacco, disease resistance improved and the degree of disease resistance increased with the expression level of CALTPI and CALTPII [55]. nsLTPs were reported to have a certain role in plant disease resistance signal pathways. Maldonado et al. found that Arabidopsis nsLTP (DIR1) was involved in the process of an important mobile signal production or transmission in leaf which was infected with Phytophthora syringae [56]. Furthermore, they proposed that DIR1 interacts with a lipid-derived molecule to promote long-distance signal transmission. Grafting wild type tobacco and CALTPI and CALTPII overexpressed tobacco showed that the CALTP participates in long-distance system signaling transmission and plays an important role in the interaction of plant pathogens by activating SAR-mediated signaling pathway [55]. The above studies showed that nsLTPs play an important role in the resistance of plants and the invasion of a variety of pathogens. To further assess possible disease resistance-related functions of BnLTPs, we compared the position of BnLTPs with known QTL for blackleg resistance [44]. The blackleg resistance QTLs contained 14 core and variable BnLTP genes. Two genes (BnLTPG.10 and BnLTPG.12) showed the lowest mutation in the LepR1 locus but had a large number of missense variants, it may be indicative that these genes were under positive selection pressure (Fig. 8B). There was a more synonymous variants that occurred on LepR1 locus compared with $R \operatorname{lm} 3$ and $R \operatorname{lm} 4$ loci, revealing that LepR1 locus is more stable than $R \operatorname{lm} 3$ and $R \operatorname{lm} 4$ loci. Identification of BnLTPs within QTL may provide a basis for Brassica napus breeding in the future. According to previous studies, CNVs (copy number variations) are major genetic variations influencing gene expression and phenotype. There are more CNVs than chromosome structural variations, and the total number of nucleotides covered by CNVs is much larger than SNP number in the whole genome $[57,58]$. Therefore, in the future research, we will analyze the impact of CNVs on 
nsLTP gene families. The characteristics and expression patterns of BnLTPs identified in this study provide more information on resistant materials for molecular breeding.

\section{Abbreviations}

nsLTPs: Non-specific lipid transfer proteinsQTL: quantitative trait locus; ECM: 8-cysteine motif; LTPs: Lipid transfer proteins; GPI: Glycosylphosphatidylinositol; DRN1: Disease-related non-specific lipid transfer protein 1; CDS: Coding sequence; CDD: Conserved domain database; GG: Gangan; ZY: Zheyou7; SL: Shengli; TA:Tapidor; QU: Quinta; WT: Westar; NO: No2127; B. napus: Brassica napus; B. oleracea: Brassica oleracea; B. rapa: Brassica rapa; ERF: Ethyleneresponsive factor; NAC: NAM, ATAF, and CUC; LBD: Lateral organ boundaries domain; R-line: Resistant line; S-line: Susceptible line; SNPs: Single nucleotide polymorphisms; PAV: Presence-absence variation; RGA: Resistance gene analog; S. sclerotiorum: Sclerotinia sclerotiorum.

\section{Supplementary Information}

The online version contains supplementary material available at https://doi. org/10.1186/s12870-021-03408-5.

\section{Additional file 1: Supplymentary file 1: The nsLTP protein set with classifications}

Additional file 2: Supplymentary Table S1: Count of BnLTP genes in Brassica napus pangenome

Additional file 3: Supplymentary Table S2: Ka/Ks analysis of tandem duplication BnLTP genes

Additional file 4: Supplymentary Table S3: Ka/Ks analysis of segmental duplication BnLTP genes

Additional file 5: Supplymentary Table S4: The differentially expression analysis results of BnLTP genes that responses to Sclerotinia sclerotiorum

Acknowledgements

We acknowledge Chunni Liu's help in this work.

\section{Authors' contributions}

Conceptualization, M.L. and Y.L.; methodology, X.K.; software, Y.H.; validation, K.C., X.K. and Y.L.; data curation, X.K.; writing — original draft preparation, Y.L. and Y.H.; writing - review and editing, M.L. All authors have read and agreed to the published version of the manuscript.

\section{Funding}

This work was supported by the Guangxi Science and Technology Base and Talent Project (Guike AD19245098), Guangxi Natural Science Foundation (2020GXNSFBA297045; 2020GXNSFBA297157), Research Project of Key Laboratory of Ecology of Rare and Endangered Species and Environmental Protection (ERESEP2020Z09), Sustainable development innovation project of Guangxi Normal University (2020CX003) and National Natural Science Foundation of China $(31871656 ; 32101684)$. The funding body were not involved in the design of the study, data collection, interpretation of data, or in writing the manuscript.

\section{Availability of data and materials}

The data presented in this study are available on request from the corresponding author. All databases used in the study are open for public access. The references of these databases are as follow: the B. napus pangenome information resource (http://cbi.hzau.edu.cn/bnapus/) [22]; TAIR (http://www.arabi dopsis.org); NCBI (https://www.ncbi.nlm.nih.gov); Binding site prediction tool (http://plantregmap.gao-lab.org/binding_site_prediction.php in plantregmap); Retrieve mapping tool (https://www.uniprot.org/uploadlists/);AgriGO (http://bioinfo.cau.edu.cn/agriGO/analysis.php); bedtools v2.16.2 intersect [33]; Known blackleg resistance-linked QTL were collected from the literature [34-38]. Waterfall plots were drawn using Variant Effect Predictor v99 [39], GenVisR v1.11.3 [40], vcftools v0.1.16 [41].

\section{Declarations}

Ethics approval and consent to participate

Not applicable.

\section{Consent for publication}

Not applicable.

\section{Competing interests}

The authors declare no conflict of interest.

\section{Author details}

${ }^{1}$ Key Laboratory of Ecology of Rare and Endangered Species and Environmental Protection, Guangxi Key Laboratory of Landscape Resources Conservation and Sustainable Utilization in Lijiang River Basin, College of Life Science, Guangxi Normal University, Guilin, China. ${ }^{2}$ School of Mechanical and Electrical Engineering, Guilin University of Electronic Technology, Guilin, China. ${ }^{3}$ Department of Biotechnology, College of Life Science and Technology, Huazhong University of Science and Technology, Wuhan, China.

Received: 4 June 2021 Accepted: 15 December 2021

Published online: 07 January 2022

\section{References}

1. Meng C, Yan Y, Liu Z, Chen L, Zhang Y, Li X, et al. Systematic analysis of cotton non-specific lipid transfer protein family revealed a special group that is involved in fiber elongation. Front Plant Sci. 2018;9:1285.

2. Boutrot F, Chantret N, Gautier MF. Genome-wide analysis of the rice and arabidopsis non-specific lipid transfer protein (nsLtp) gene families and identification of wheat nsLtp genes by EST data mining. BMC Genomics. 2008:9:1-19.

3. Edstam MM, Viitanen L, Salminen TA, Edqvist J. Evolutionary history of the non-specific lipid transfer proteins. Mol Plant. 2011:4:947-64.

4. Scheurer S, Lauer I, Foetisch K, Moncin MSM, Retzek M, Hartz C, et al. Strong allergenicity of Pru av 3, the lipid transfer protein from cherry, is related to high stability against thermal processing and digestion. J Allergy Clin Immunol. 2004;114:900-7.

5. Odintsova TI, Slezina MP, Istomina EA, Korostyleva TV, Kovtun AS, Kasianov AS, et al. Non-specific lipid transfer proteins in Triticum kiharae dorof. Et migush:: identification, characterization and expression profiling in response to pathogens and resistance inducers. Pathogens. 2019;8:221.

6. Li J, Gao G, Xu K, Chen B, Yan G, Li F, et al. Genome-wide survey and expression analysis of the putative non-specific lipid transfer proteins in Brassica rapa L. PLoS One. 2014:9:e84556.

7. Sabala I, Elfstrand M, Farbos I, Clapham D, Von Arnold S. Tissue-specific expression of $\mathrm{Pa}$ 18, a putative lipid transfer protein gene, during embryo development in Norway spruce (Picea abies). Plant Mol Biol. 2000:42:461-78.

8. Eklund DM, Edqvist J. Localization of nonspecific lipid transfer proteins correlate with programmed cell death responses during endosperm degradation in Euphorbia lagascae seedlings. Plant Physiol. 2003;132:1249-59.

9. Park SY, Jauh GY, Mollet JC, Eckard KJ, Nothnagel EA, Walling LL, et al. A lipid transfer-like protein is necessary for lily pollen tube adhesion to an in vitro stylar matrix. Plant Cell. 2000;12:151-63.

10. Nieuwland J, Feron R, Huisman BAH, Fasolino A, Hilbers CW, Derksen J, et al. Lipid transfer proteins enhance cell wall extension in tobacco. Plant Cell. 2005;17:2009-19.

11. Zhao J, Wang S, Qin J, Sun C, Liu F. The lipid transfer protein OsLTPL159 is involved in cold tolerance at the early seedling stage in rice. Plant Biotechnol J. 2020;18:756-69.

12. Gangadhar BH, Sajeesh K, Venkatesh J, Baskar V, Abhinandan K, Yu JW, et al. Enhanced tolerance of transgenic potato plants over-expressing non-specific lipid transfer protein-1 (StnsLTP1) against multiple abiotic stresses. Front Plant Sci. 2016;7:1228.

13. Liu F, Xiong X, Wu L, Fu D, Hayward A, Zeng X, et al. BraLTP1, a lipid transfer protein gene involved in epicuticular wax deposition, cell proliferation and flower development in Brassica napus. PLoS One. 2014:9:e110272. 
14. Tian N, Liu F, Wang P, Yan X, Gao H, Zeng X, et al. Overexpression of BraLTP2, a lipid transfer protein of brassica napus, results in increased trichome density and altered concentration of secondary metabolites. Int J Mol Sci. 2018;19:1733.

15. Chalhoub B, Denoeud F, Liu S, Parkin IAP, Tang H, Wang X, et al. Early allopolyploid evolution in the post-neolithic Brassica napus oilseed genome. Science (80- ). 2014;345:950-3.

16. Sun F, Fan G, Hu Q, Zhou Y, Guan M, Tong C, et al. The high-quality genome of Brassica napus cultivar 'ZS11' reveals the introgression history in semi-winter morphotype. Plant J. 2017;92:452-68.

17. Li YH, Zhou G, Ma J, Jiang W, Jin LG, Zhang Z, et al. De novo assembly of soybean wild relatives for pan-genome analysis of diversity and agronomic traits. Nat Biotechnol. 2014;32:1045-52.

18. Lu F, Romay MC, Glaubitz JC, Bradbury PJ, Elshire RJ, Wang T, et al. Highresolution genetic mapping of maize pan-genome sequence anchors. Nat Commun. 2015;6:1-8.

19. Wang W, Mauleon R, Hu Z, Chebotarov D, Tai S, Wu Z, et al. Genomic variation in 3,010 diverse accessions of Asian cultivated rice. Nature. 2018;557:43-9.

20. Zhao Q, Feng Q, Lu H, Li Y, Wang A, Tian Q, et al. Pan-genome analysis highlights the extent of genomic variation in cultivated and wild rice. Nat Genet. 2018;50:278-84.

21. Gao L, Gonda I, Sun H, Ma Q, Bao K, Tieman DM, et al. The tomato pangenome uncovers new genes and a rare allele regulating fruit flavor. Nat Genet. 2019;51:1044-51.

22. Song JM, Guan Z, Hu J, Guo C, Yang Z, Wang S, et al. Eight high-quality genomes reveal pan-genome architecture and ecotype differentiation of Brassica napus. Nat Plants. 2020;6:34-45.

23. Song JM, Liu DX, Xie WZ, Yang Z, Guo L, Liu K, et al. BnPIR: Brassica napus pan-genome information resource for 1689 accessions. Plant Biotechnol J. 2021;19:412-4.

24. Pertea G, Pertea M. GFF Utilities: GffRead and GffCompare. F1000Research. 2020:9:304

25. El-Gebali S, Mistry J, Bateman A, Eddy SR, Luciani A, Potter SC, et al. The Pfam protein families database in 2019. Nucleic Acids Res. 2019;47:D427-32

26. Lu S, Wang J, Chitsaz F, Derbyshire MK, Geer RC, Gonzales NR, et al. CDD/ SPARCLE: the conserved domain database in 2020. Nucleic Acids Res. 2020;48:D265-8.

27. Nielsen H. Predicting secretory proteins with signalP. Methods Mol Biol. 2017;1611:59-73.

28. Bailey TL, Boden M, Buske FA, Frith M, Grant CE, Clementi L, et al. MEME suite: tools for motif discovery and searching. Nucleic Acids Res. 2009;37:W202-8.

29. Hu B, Jin J, Guo AY, Zhang H, Luo J, Gao G. GSDS 2.0: an upgraded gene feature visualization server. Bioinformatics. 2015:31:1296-7.

30. Cannon SB, Mitra A, Baumgarten A, Young ND, May G. The roles of segmental and tandem gene duplication in the evolution of large gene families in Arabidopsis thaliana. BMC Plant Biol. 2004:4:1-21.

31. Chen C, Chen H, Zhang Y, Thomas H, Frank M, He Y, et al. TBtools - an integrative toolkit developed for interactive analyses of big biological data. bioRxiv. 2018;13:1194-202.

32. Love Ml, Huber W, Anders S. Moderated estimation of fold change and dispersion for RNA-seq data with DESeq2. Genome Biol. 2014;15:1-21.

33. Quinlan AR, Hall IM. BEDTools: a flexible suite of utilities for comparing genomic features. Bioinformatics. 2010;26:841-2.

34. Delourme R, Pilet-Nayel ML, Archipiano M, Horvais R, Tanguy X, Rouxel $T$, et al. A cluster of major specific resistance genes to Leptosphaeria maculans in Brassica napus. Phytopathology. 2004;94:578-83.

35. Larkan NJ, Raman H, Lydiate DJ, Robinson SJ, Yu F, Barbulescu DM, et al. Multi-environment QTL studies suggest a role for cysteine-rich protein kinase genes in quantitative resistance to blackleg disease in Brassica napus. BMC Plant Biol. 2016;16:1-16

36. Leflon M, Brun $H$, Eber F, Delourme R, Lucas MO, Vallée $P$, et al. Detection, introgression and localization of genes conferring specific resistance to Leptosphaeria maculans from Brassica rapa into B. napus. Theor Appl Genet. 2007;115:897-906.

37. Raman R, Taylor B, Lindbeck K, Coombes N, Barbulescu D, Salisbury P, et al. Molecular mapping and validation of RIm1 gene for resistance to Leptosphaeria maculans in canola (Brassica napus L.). Crop Pasture Sci. 2012:63:1007-17.
38. Raman R, Taylor B, Marcroft S, Stiller J, Eckermann P, Coombes N, et al. Molecular mapping of qualitative and quantitative loci for resistance to Leptosphaeria maculans causing blackleg disease in canola (Brassica napus L.). Theor Appl Genet. 2012;125:405-18.

39. McLaren W, Gil L, Hunt SE, Riat HS, Ritchie GRS, Thormann A, et al. The Ensembl variant effect predictor. Genome Biol. 2016;17:1-14.

40. Skidmore ZL, Wagner AH, Lesurf R, Campbell KM, Kunisaki J, Griffith OL, et al. GenVisR: genomic visualizations in R. Bioinformatics. 2016;32:3012-4.

41. Danecek P, Auton A, Abecasis G, Albers CA, Banks E, DePristo MA, et al. The variant call format and VCFtools. Bioinformatics. 2011;27:2156-8.

42. Wang W, Gao T, Chen J, Yang J, Huang H, Yu Y. The late embryogenesis abundant gene family in tea plant (Camellia sinensis): genome-wide characterization and expression analysis in response to cold and dehydration stress. Plant Physiol Biochem. 2019;135:277-86.

43. Wu J, Zhao Q, Yang Q, Liu H, Li Q, Yi X, et al. Comparative transcriptomic analysis uncovers the complex genetic network for resistance to Sclerotinia sclerotiorum in Brassica napus. Sci Rep. 2016;6:1-16.

44. Dolatabadian A, Bayer PE, Tirnaz S, Hurgobin B, Edwards D, Batley J. Characterization of disease resistance genes in the Brassica napus pangenome reveals significant structural variation. Plant Biotechnol J. 2020;18:969-82.

45. Hurgobin B, Golicz AA, Bayer PE, Chan CKK, Tirnaz S, Dolatabadian A, et al. Homoeologous exchange is a major cause of gene presence/absence variation in the amphidiploid Brassica napus. Plant Biotechnol J. 2018;16:1265-74.

46. Bayer PE, Golicz AA, Tirnaz S, Chan CKK, Edwards D, Batley J. Variation in abundance of predicted resistance genes in the Brassica oleracea pangenome. Plant Biotechnol J. 2019;17:789-800.

47. Li F, Fan K, Ma F, Yue E, Bibi N, Wang M, et al. Genomic identification and comparative expansion analysis of the non-specific lipid transfer protein gene family in Gossypium. Sci Rep. 2016;6:1-15.

48. Dhar N, Caruana J, Erdem I, Raina R. An Arabidopsis DISEASE RELATED NONSPECIFIC LIPID TRANSFER PROTEIN 1 is required for resistance against various phytopathogens and tolerance to salt stress. Gene. 2020;753:144802

49. McLaughlin JE, Bin-Umer MA, Widiez T, Finn D, McCormick S, Tumer NE. A lipid transfer protein increases the glutathione content and enhances Arabidopsis resistance to a trichothecene mycotoxin. PLoS One. 2015;10:e0130204.

50. Patkar RN, Chattoo BB. Transgenic indica rice expressing ns-LTP-like protein shows enhanced resistance to both fungal and bacterial pathogens. Mol Breed. 2006;17:159-71.

51. Isaac Kirubakaran S, Begum SM, Ulaganathan K, Sakthivel N. Characterization of a new antifungal lipid transfer protein from wheat. Plant Physiol Biochem. 2008;46:918-27

52. Lee SB, Go YS, Bae HJ, Park JH, Cho SH, Cho HJ, et al. Disruption of glycosylphosphatidylinositol-anchored lipid transfer protein gene altered cuticular lipid composition, increased plastoglobules, and enhanced susceptibility to infection by the fungal pathogen alternaria brassicicola. Plant Physiol. 2009;150:42-54.

53. Ho WJ, Ki DK, Byung KH. Identification of pathogen-responsive regions in the promoter of a pepper lipid transfer protein gene (CALTPI) and the enhanced resistance of the CALTPI transgenic Arabidopsis against pathogen and environmental stresses. Planta. 2005;221:361-73.

54. Jung HW, Kim W, Hwang BK. Three pathogen-inducible genes encoding lipid transfer protein from pepper are differentially activated by pathogens, abiotic, and environmental stresses. Plant Cell Environ. 2003;26:915-28.

55. Sarowar S, Kim YJ, Kim KD, Hwang BK, Ok SH, Shin JS. Overexpression of lipid transfer protein (LTP) genes enhances resistance to plant pathogens and LTP functions in long-distance systemic signaling in tobacco. Plant Cell Rep. 2009;28:419-27.

56. Maldonado AM, Doerner P, Dixonk RA, Lamb CJ, Cameron RK. A putative lipid transfer protein involved in systemic resistance signalling in Arabidopsis. Nature. 2002;419:399-403.

57. Lupski JR. Genomic rearrangements and sporadic disease. Nat Genet. 2007;39:S43-6.

58. Yu P, Wang $\mathrm{CH}, \mathrm{Xu} \mathrm{Q}$, et al. Genome-wide copy number variations in Oryza sativa L[J]. BMC genomics. 2013:14(1):1-12.

\section{Publisher's Note}

Springer Nature remains neutral with regard to jurisdictional claims in published maps and institutional affiliations. 